\title{
Distaler Femurersatz nach suprakondylärer periprothetischer Femurfraktur
}

\author{
Frieder Schulz
}

Jährlich werden in Deutschland ca. 75000 Knieendoprothesen implantiert und entsprechend der demografischen Entwicklung ist mit einem weiteren Anwachsen der Implantationszahlen zu rechnen [1]. Als Folge wird der Unfallchirurg immer häufiger mit periprothetischen Frakturen konfrontiert werden. Die Therapie dieser Frakturen ist problematisch, denn es müssen nicht nur die allgemeinen und spezifischen lokalen Risikofaktoren, der Frakturtyp und die meist vorliegende Osteoporose, sondern auch das Prothesendesign sowie der Funktionszustand der Prothese Berücksichtigung finden. Häufig erschweren auch in der Nachbarschaft einliegende Hüftendoprothesen das operative Vorgehen. Entscheidend für die Planung der Operation ist neben der Frakturklassifikation die vorbestehende Funktion der Knieprothese. $\mathrm{Zu}$ fahnden ist nach Zeichen einer Lockerung, Instabilität und Achsfehlstellung. Für die periprothetischen kniegelenksnahen Frakturen ist eine eigene Klassifikation entwickelt worden, die eine Entscheidung für den zu wählenden operativen Weg ermöglicht [3]. Bei Typ-I-Frakturen verläuft die Fraktur oberhalb der Femurkondylenprothese, bei Typ-II-Frakturen am Oberrand der Kondylenkappe beginnend und nach proximal auslaufend. Bei Typ-III-Frakturen liegt die Bruchlinie unterhalb des Oberrands der Femurkomponente. Liegt ein unauffälliger und fester Sitz der Knieprothese vor, ist die Indikation zur Stabilisierung der Fraktur mit einem winkelstabilen Plattensystem (z.B. LISS) bzw. bei offener Femurkondylenkomponente auch mit einem retrograden Nagel gegeben [4]. Bei kleinem distalen Fragment und geschlossener Box der Femurkomponente mit nicht möglicher extramedullärer Stabilisierung verbleibt der Pro-

OP-JOURNAL 2008; 24: 234-235

(c) Georg Thieme Verlag KG Stuttgart • New York DOI 10.1055/s-2008-1039054
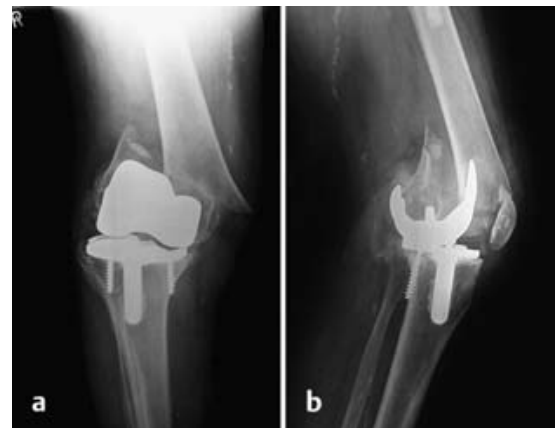

Abb. $1 \mathbf{a}$ und $\mathbf{b}$ Röntgenologische Situation bei Klinikaufnahme, dislozierte suprakondyläre periprothetische Femurfraktur Typ II bei einliegender Performance-Knie-TEP mit sichtbarem Verschleiß.

thesenwechsel mit einem Knie-Rekonstruktionsimplantat. Ist die Knieprothese gelockert, sollte in jedem Fall der Komponentenwechsel mit intramedullärer Schaftstabilisierung erfolgen. Ausnahmsweise ist ein zweizeitiges Vorgehen angesagt, d. h. dass vor dem erforderlichen Wechsel einer gelockerten Prothese eine Osteosynthese Anwendung findet und erst nach Heilung der Fraktur der Prothesenwechsel durchgeführt wird [2]. Bei einem solchen Vorgehen ist mit einem Fehlverlauf der Osteosynthese zu rechnen.

\section{Falldarstellung}

Die 74-jährige Patientin war in ihrer Wohnung mit dem rechten Bein hängen geblieben, auf das rechte Kniegelenk gestürzt und wurde bei 12 Jahre einliegender Performance-Knieprothese mit einer suprakondylären periprothetischen Femurfraktur in die Klinik eingewiesen (Abb.1).

Die anderen großen Gelenke der unteren Extremitäten waren ebenfalls endoprothetisch ersetzt (Hüft-TEP links 1992, Knie-TEP links 1999, Hüft-TEP rechts 2001). Wegen der multiplen Nebenerkrankungen der $166 \mathrm{~cm}$ großen und $90 \mathrm{~kg}$ schweren Patientin (CIHK, arte-

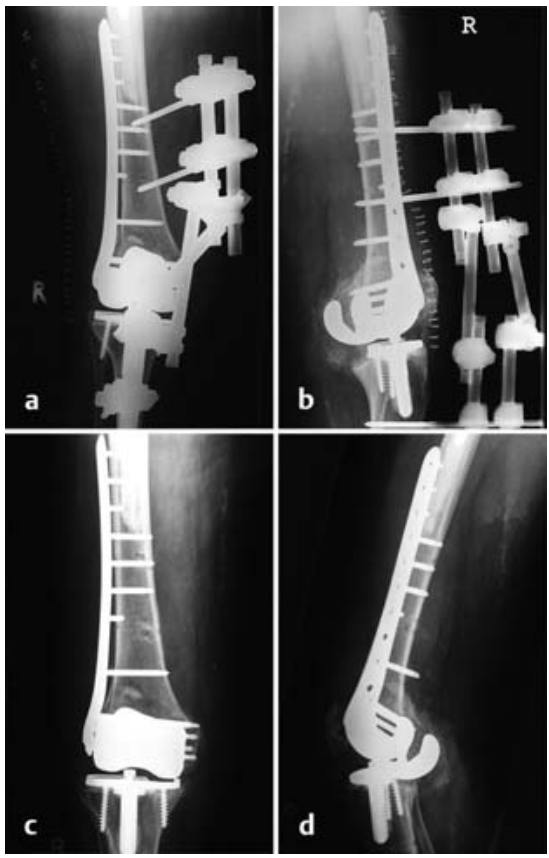

Abb. $\mathbf{2}$ a bis $\mathbf{d}$ a, b LISS-Osteosynthese und protektiver Fixateur externe. c, d Situation nach Entfernung des Fixateurs 6 Wochen postoperativ.

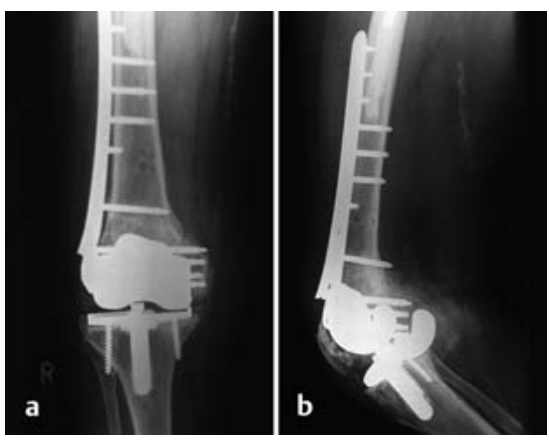

Abb. $3 \mathbf{a}$ und $\mathbf{b}$ Röntgenbilder 1 Jahr postoperativ, Bruch der LISS-Platte.

rielle Hypertonie, paVK IIa, COPD, Diabetes mellitus mit Polyneuropathie, kompensierte Niereninsuffizienz, postthrombotisches Syndrom beider Unterschenkel) und des aktuellen Quick-Wertes $<30 \%$ bei Falithrombehandlung erfolgte am Unfalltag die notfallmäßige 

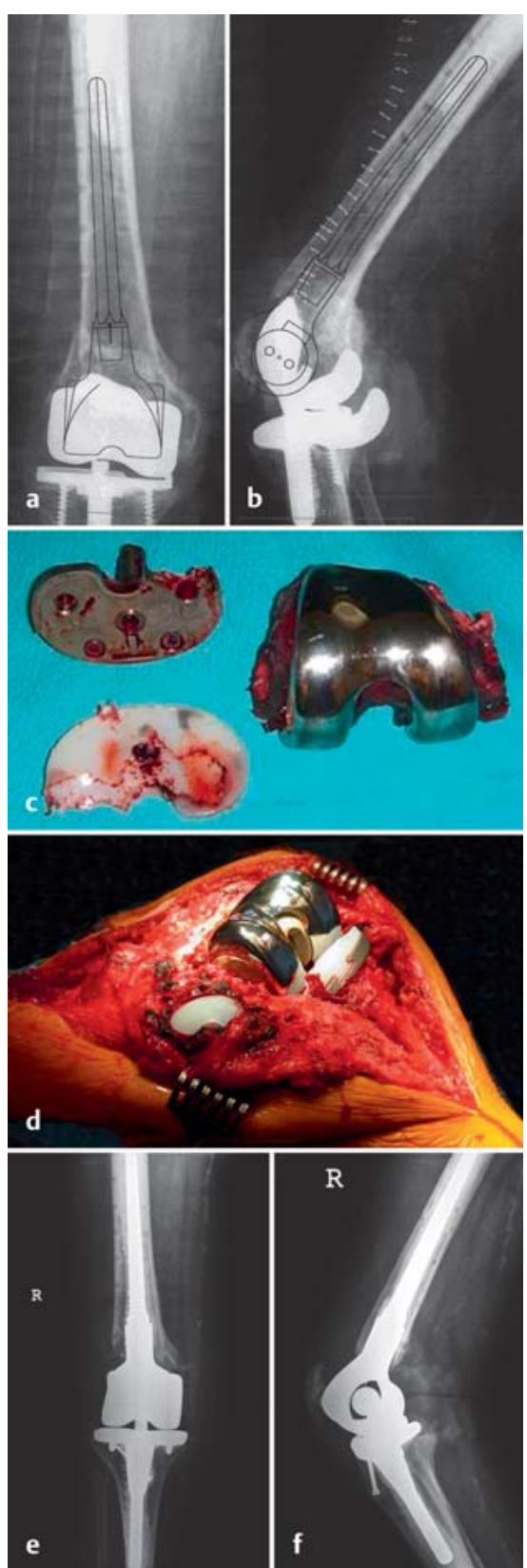

Abb. 4 a bis $\mathbf{f}$ a, b Planungsskizzen. c, $\mathbf{d}$ Explantierte Performance-Prothese mit distalem Frakturfragment; einliegendes Knie-Rekonstruktionsimplantat - intraoperativer Situs. e, f Postoperative Röntgenbilder.

Stabilisierung der Fraktur mit einem gelenküberbrückenden Fixateur externe und erst 3 Tage später die definitive Versorgung mit einer LISS-Platte (Abb. 2).

Die primären Röntgenaufnahmen zeigten deutliche Verschleißerscheinungen der tibialen Prothesenkomponente, sodass auch der Prothesenwechsel mit intramedullärer Schaftstabilisierung erwogen wurde. Wegen des festen Sitzes der Femurkondylenkappe, der zu erwar-
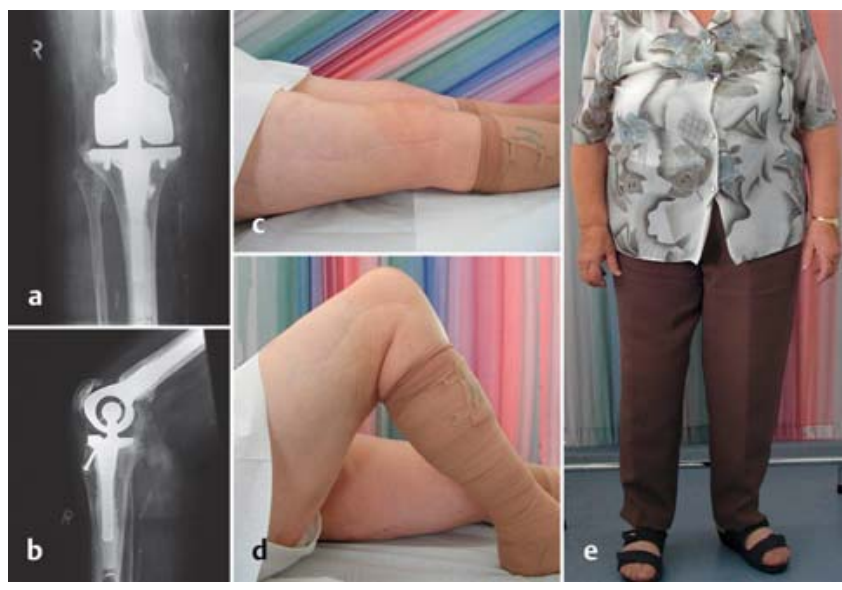

Abb. 5 a bis e

a,b Röntgenkontrollaufnahmen 2 Jahre postoperativ. c-e Funktionsaufnahmen nach 2 Jahren.

tenden Schädigung des distalen Fragments bei der Explantation, der Multimorbidität der Patientin und weil die allerdings nicht klagsame Frau vor dem Sturzereignis mit der Knieprothese nach ihrer Aussage „zurechtgekommen“ war, fiel die Entscheidung zur Frakturstabilisierung mit einer LISS-Platte. Vorübergehend wurde zur Mobilisierbarkeit der Patientin - da sie das rechte Bein nicht ausreichend entlasten konnte - und wegen der erst- bis zweitgradigen medialen Knieinstabilität für 6 Wochen ein protektiver Fixateur externe angelegt. Nach Entfernung desselben wurde die Vollbelastung des rechten Beines mit einer stabilisierenden Kniegelenksorthese ohne Limitierung des Bewegungsausmaßes erlaubt. Nach einem Jahr erfolgte die Wiedereinweisung der Patientin in unsere Klinik wegen akuter Schmerzen im rechten Oberschenkel und erneuter Belastungsinsuffizienz des rechten Beines ohne äußere Einwirkung. Die Röntgendiagnostik ergab den Bruch der LISS-Platte in Höhe der Fraktur, die Fraktur war nicht konsolidiert (Abb. 3).

Die insuffiziente instabile Knieprothese hat die Frakturheilung verhindert und zum Ermüdungsbruch der Platte geführt. Der Prothesenwechsel war jetzt nicht mehr zu umgehen. Nach Entfernung der gebrochenen Platte wurde aufgrund der Größe des distalen Frakturfragments und der zu erwartenden Schädigung desselben bei der Explantation der distale Femurersatz mit einem gekoppelten Knie-Rekonstruktionsimplantat (Implantcast) geplant und durchgeführt (Abb.4).

Nach 4-wöchigem stationären Aufenthalt und komplikationslosem postoperativen Verlauf war die Patientin am reziproken Gehgestell mobilisiert und konnte zur geriatrischen Rehabilitation verlegt werden. Bei der Nachuntersuchung 2 Jahre postoperativ ist das Gehen unter voller Belastung des rechten Beines schmerzfrei, das Kniegelenk stabil. Das Bewegungsausmaß beträgt Extension/Flexion 0/0/110 . Kein Gelenkerguss, allerdings noch deutliche Quadrizepsmuskelatrophie. Die Röntgenaufnahmen zeigen das regelrecht einliegende Knie-Rekonstruktionsimplantat ohne Lockerungszeichen (Abb. 5).

Fazit

Die vorliegende Kasuistik verdeutlicht anhand eines Fehlverlaufs die Schwierigkeiten der Versorgung periprothetischer kniegelenksnaher Frakturen. Es wird gezeigt, dass bei Insuffizienz der einliegenden Knieprothese trotz korrekter Osteosynthese der periprothetischen Fraktur keine Heilung erfolgen kann. Der Zustand der Prothese muss als entscheidender Faktor bei der operativen Planung Berücksichtigung finden. Bei Lockerung oder Verschleiß der Prothese ist bei der Stabilisierung der Fraktur in jedem Fall der Wechsel der Prothese durchzuführen, bei Typ-IIIFrakturen und festsitzender Femurkomponente sollte der distale Femurersatz in Erwägung gezogen werden.

\section{Literatur beim Verfasser}

\section{Priv.-Doz. Dr. med. Frieder Schulz}

Chefarzt

\section{Klinik für Unfall- und}

Wiederherstellungschirurgie

Klinikum Weimar

Henry-van-de-Velde-Straße 2

99425 Weimar

E-Mail: trauma@klinikum-weimar.de 